\title{
Crecimiento agrario y crisis/transformación ambiental en la corona de Castilla (siglos XV-XVI)
}

\author{
Agrarian growth and environmental crisis/transformation in the crown \\ of Castile (15-16 centuries)
}

Julián CLEMENTE RAMOS

\begin{abstract}
Author:
Julián Clemente Ramos

Catedrático de Historia Medieval. Universidad de Extremadura (Cáceres, Spain) clemente@unex.es

https://orcid.org/0000-0001-8900-9754

Date of reception: 16/04/21

Date of acceptance: 19/05/21

Citation:

Clemente Ramos, J. (2021). Crecimiento agrario y crisis/transformación ambiental en la corona de Castilla (siglos XV-XVI). Anales de la Universidad de Alicante. Historia Medieval, (22), 57-79.

https://doi.org/10.14198/medieval.19596

Funding: Grupo HUM023 (Junta de Extremadura). La elaboración de este trabajo se ha beneficiado de fondos destinados a los grupos de investigación por la Junta de Extremadura (Consejería de Economía e Infraestructuras; financiado con fondos FEDER) para el periodo 2018-2021.
\end{abstract}

(C) 2021 Julián Clemente Ramos

Licence: This work is licensed under a Creative Commons Attribution 4.0 International License (CC BY 4.0).

\section{RESUMEN}

El crecimiento demográfico fue intenso y sostenido en la corona de Castilla durante los siglos XV y XVI. Las zonas meridionales, tardía e insuficientemente ocupadas, van a alcanzar niveles poblacionales relativamente importantes. Esta nueva realidad tendrá efectos intensos en una economía de carácter extensivo. Una población en aumento va a generar un sensible aumento del terrazgo cerealista y la definitiva agrarización del espacio. Este proceso de antropización afectará de modo sensible a la vegetación natural. El bosque sufre un claro retroceso. Frente a la situación anterior de abundancia se empieza a percibir como un recurso escaso y sobre todo limitado. Las ordenanzas de montes muestran la necesidad de desarrollar una explotación sostenible y regularán estrictamente su aprovechamiento. Estos cambios incidirán de modo sensible sobre la fauna salvaje y la caza. Los grandes herbívoros, necesitados de una abundante cubierta forestal, se verán especialmente afectados. El lobo, ante la reducción de sus fuentes de alimentación, se convertirá en una clara amenaza para la ganadería. La lucha contra este animal, que comienza ahora de modo sistemático, es un indicador de las transformaciones y los desequilibrios ambientales.

PALABRAS CLAVE: baja Edad Media; siglo XVI; historia ambiental; monte; fauna; lobo. 


\section{ABSTRACT}

The demographic growth was intense and sustained in the crown of Castile during the fifteenth and sixteenth centuries. The late and under-occupied southern areas reached relatively significant population levels. This new reality had an intense effect on an extensive economy. A growing population generated a significant increase in cereal land and the definitive agrarization of the space. This process of anthropization significantly affected natural vegetation. The forest suffered a clear setback. Faced with the previous situation of abundance, it was beginning to be perceived as a scarce and above all limited resource. Forest ordinances show the need to develop sustainable exploitation and strictly regulated its use. These changes had a significant impact on wildlife and hunting. Large herbivores, in need of abundant forest cover, will be particularly affected. The wolf, faced with the reduction of its food sources, became a clear threat to livestock. The fight against this animal, which was in that moment when began systematically, is an indicator of environmental transformations and imbalances.

KEYWORDS: Later Middle Ages; sixteenth century; environmental history; forest; wildlife; wolf.

\section{INTRODUCCIÓN}

Los estudios ambientales forman parte de las preocupaciones del mundo actual por razones que no es necesario explicar. No sorprende, por ello, que esta temática haya generado un interés creciente en el ámbito de la historia. El medievalismo de la corona de Castilla ha mostrado, sin embargo, una atención limitada. De hecho, la mayor parte de la información disponible sobre la materia se encuentra en trabajos que no presentan un interés específico en la problemática ambiental/ecológica.

La historia ambiental se ha estado haciendo realmente antes del desarrollo de esta línea. La historia rural ha estado suministrando una información valiosa. Una roza, un adehesamiento o una captura cinegética nos revelan partes de un todo, al margen de que el interés pueda centrarse en la producción cerealista, los pastos o la caza. Los modelos ecológicos integran todos los elementos de una realidad de forma integrada e interrelacionada. Una buena compresión de la problemática ambiental exige una metodología precisa y clara que permita entender el funcionamiento de un ecosistema, entendido en su globalidad, y de sus cambios y desequilibrios. La historia rural, además, entendemos que no puede abordarse adecuadamente sin una perspectiva ecológica, puesto que el hombre destruye, cambia y, sobre todo, adapta los diversos espacios para ajustarlos a sus necesidades.

La Edad Media es un periodo de una profunda transformación ambiental en el occidente europeo. En el milenio que transcurre entre los años 500 y 1500, se asiste a una antropización creciente del paisaje debido al crecimiento demográfico, la 
agrarización de la economía rural y el control cada vez más estricto de los espacios de pastos. Esta evolución afectó profundamente a la vegetación natural y, consiguientemente, a la fauna salvaje. Todo esto no puede explicarse si no consideramos que el crecimiento medieval es fundamental y casi exclusivamente extensivo. Las sociedades precapitalistas o tradicionales no tienen capacidad para incrementar la productividad del trabajo al ritmo del crecimiento demográfico. Su crecimiento es, en términos anuales, tendencialmente cero (lo cual no significa que no se registren cambios, mal comprendidos, a medio y sobre todo largo plazo). Las crecientes necesidades alimenticias se obtienen con la ampliación del terrazgo agrario y específicamente cerealista por la necesidad de producir más calorías por unidad espacial. Se produce, de este modo, un claro cambio paisajístico. De modo creciente, el paisaje se ajusta de forma intensa y creciente a las necesidades humanas. Este proceso afecta de modo sensible al bosque y la fauna.

En el presente artículo intentaremos relacionar la problemática ambiental con el crecimiento demográfico y agrario que se registra en Castilla entre las primeras décadas del siglo XV y finales del XVI. Nos centraremos fundamentalmente en la meseta y zonas meridionales. Debemos precisar que no pretendemos abordar ningún estado de la cuestión, algo totalmente fuera de nuestras intenciones, sino una explicación sistémica de los cambios y desequilibrios ambientales y de sus causas. Esto nos permitirá no ser exhaustivos en la información suministrada y elegir los entornos espaciales (las zonas meridionales, la meseta y especialmente Extremadura) más cercanos o sobre los que hemos centrado nuestra investigación.

\section{CRECIMIENTO DEMOGRÁFICO Y AGRARIZACIÓN DEL ESPACIO}

La corona de Castilla, y específicamente las zonas meridionales (para la baja Edad Media, el sur del Sistema Central), desarrolló más tardíamente que otras zonas europeas intensamente ocupadas hacia 1300 el proceso de agrarización del espacio․ El espacio situado al sur del Tajo sólo se controla de modo definitivo después de las Navas de Tolosa (1212). La rápida expansión castellana subsiguiente, que supuso un aumento territorial en torno al $50 \%$, y la amplia limpieza étnica sólo permitió una ocupación lenta y desigual que estaba muy lejos de alcanzar su madurez cuando las pestes bajomedievales rompieron una clara dinámica de crecimiento. Con excepciones, las zonas meridionales sólo dispondrán de una ocupación relativamente madura, dentro de los modelos y formas de explotación imperantes, en el siglo XV.

Desde las primeras décadas del siglo XV y hasta las últimas del XVI, periodo raramente abordado de modo unitario por razones académicas, se desarrolla un

1 Un ejemplo claro y preciso en Rösener, 1990, p. 49, fig. 1 -mapas de la región del Diemel hacia los años 500 y $1290-$ (el mapa es de H. Jäger). 
crecimiento demográfico sostenido en Castilla. Los espacios meridionales pasan a estar suficiente o densamente ocupados y a presentar conflictos derivados de esta situación. El motor ineludiblemente, por las razones indicadas, es la población. Los datos demográficos, tanto cuantitativos como cualitativos, son relativamente abundantes. La población en muchos casos se duplicó. En otros, menos, no alcanzó este punto, pero en muchos más lo superó.

El crecimiento demográfico, que comienza en el primer cuarto del siglo XV, adquiere fuerza en la segunda mitad. Los datos disponibles indican un crecimiento muy fuerte entre principios del XV, o antes, y finales del XVI. Los sexmos sorianos presentan incrementos de entre el 175,21 y el $297,81 \%$ para el periodo $1352-1527$ (Asenjo, 1999, p. 201). Baeza contaba con mil vecinos en 1368, 1.774 en 1407 y 6.798 en 1588 (Rodríguez Molina, 1978, p. 134). Sevilla pasa de 2.613 vecinos en 1384 a rondar los diez mil en 1533 (Collantes de Terán, 1984, pp. 156 y 169-170). La tierra de Plasencia, sin la ciudad, albergaba 1.546 vecinos en 1395 y 3.675 en 1494 (Santos Canalejo, 1986, p. 100). La información de carácter cualitativo nos permite constatar que ese incremento es sostenido y generalizado. Este crecimiento se mantuvo hasta las últimas décadas del siglo XVI. Retengamos sólo algunos datos. Entre 1530 y 1591, la población española creció un $41,2 \%$, a un ritmo medio acumulativo del 0,57 \%, que se eleva para la corona de Castilla al 0,59\%. Extremadura alcanza en 1550 una densidad de 9 habs. $/ \mathrm{km}^{2}$ que no volverá a tener hasta la década de 1830 (Marcos Martín, 2000, p. 322; Blanco Carrasco, 1999, p. 238). Este incremento demográfico, especialmente fuerte en el sur, rompe con el contraste poblacional que presentaban estas regiones y las del norte del Sistema Central hacia 1400. En torno a 1492, cuando este proceso de crecimiento no se había agotado, las zonas meridionales han alcanzado una densidad demográfica no muy alejada de la media a pesar de la importancia de formas de explotación menos intensivas como las dehesas ${ }^{2}$.

La población no es, en las sociedades precapitalista o tradicionales, un factor autónomo. Una mayor población necesita más alimentos, que es tanto como decir más pan y necesariamente más espacio para el cereal. Consiguientemente, se inicia un proceso de roturación intenso y sostenido a lo largo de un siglo y medio largo. Asistimos de este modo a una definitiva agrarización del espacio que va a ocasionar una profunda transformación ambiental. Pasamos de un paisaje dominado por el monte, la vegetación natural y los espacios de pastos a otro con una presencia mucho más importante de la agricultura.

Algunas informaciones presentan el dominio de la vegetación natural y el monte antes del siglo XV. La imagen de la Extremadura histórica como una "terram de ursorum" a principios del siglo XII nos muestra una escasa transformación ambiental y

2 Ladero Quesada, 1978, p. 31 (sus datos permiten calcular una densidad para la corona de Castilla de 11,17 habs./km² y de 9-10, o cercana a esta cantidad, para Galicia, Asturias, Andalucía, Extremadura, reino de Toledo o Murcia). 
un importante componente forestal (Clement, 1993, p. 62). Las fórmulas notariales de donación de términos en Extremadura registran una escasa transformación del paisaje vegetal en los siglos XIII-XIV (Clemente Ramos, 2001, p. 34). Los mismos deslindes presentan claras diferencias antes y después del 1400. Los accidentes geográficos son sustituidos en el siglo XV por mojones de piedras y árboles que denotan un estricto control del espacio (Clemente Ramos, 2003). Esta situación inicial, con una escasa agrarización, es compatible con un aprovechamiento de cierta entidad. El mapa del oso en Extremadura a mediados del siglo XIV refleja como este animal empieza a sufrir la competencia por el espacio. Animal muy sensible a la presencia humana, ha quedado relegado en este momento a las zonas de orografía más accidentada y montañosa (Clemente Ramos, 2015, p. 566).

El incremento del terrazgo agrario tuvo una intensidad considerable y produjo un notable impacto paisajístico. Los diezmos sevillanos en el siglo XV registran un importante crecimiento. En el primer cuarto de siglo XV, la producción cerealista se sitúa entre seiscientas y menos de ochocientas mil fanegas, con un pico en 1425, y en el tercer cuarto y la ultima década del siglo, en torno al millón y medio, con oscilaciones, y los dos millones (Ladero Quesada y González Jiménez, 1979, pp. 45 y 77). El crecimiento se mantuvo en el siglo XVI. En diversas zonas castellanas (Segovia, Zamora, Palencia, Valladolid, etc.) o la Bureba la producción cerealista alcanza su nivel más alto en la década de 1580 ó poco más tarde (Marcos Martín, 2000, pp. 351-2). Las rozas continúan en las dehesas del Campo de Montiel durante prácticamente todo el siglo. En la zona castellano-manchega, también hay un pico hacia 1580 (Fernández Petrement, 1988, pp. 99-103; Marcos Martín, 200, p. 355).

De modo general, se empieza a percibir una cierta presión sobre los recursos. Un documento de Piedrahíta alude, de modo significativo y creemos que paradigmático, a las "muchas estrechuras, a causa de la multiplicaçion de las gentes e los ganados" (Martín Martín, 1990, p. 38) ${ }^{3}$. En Huete, las parcelas yermas son raras en el siglo XV y los campesinos han ocupado "el espacio que se considera arable sin dejar apenas superficies a sus espaldas" (Sánchez Benito, 1999, p. 536). En el maestrazgo santiaguista murciano, las roturaciones se realizan a partir de 1525 sobre zonas alejadas, con orografía accidentada y suelos de baja calidad (Rodríguez Llopis, 1984, pp. 2123). Enrique Díez Sanz señala como, en Soria, desde las primeras décadas del siglo XVI "el equilibrio se rompe a favor de la agricultura", la población presiona "sobre los recursos" y se debe implementar "un nuevo sistema que haga posible que un mayor número de personas puedan vivir en un mismo territorio"( Díez Sanz, 1995, p. 327).

El crecimiento del espacio agrario ha incidido de modo global sobre el paisaje y la economía rural. Los indicios se acumulan en la submeseta norte. Se empieza a limitar el número de cabezas que puede tener cada propietario. Esto se produce

3 Este autor no deja de precisar que "se generaliza en los territorios que observamos, quizá por primera vez en su historia, la idea de escasez de tierra". 
en Paredes de Nava, donde se desarrolla una trashumancia veraniega, en 1529; en Burgos y otras pequeñas localidades de la zona entre 1458 y 1524; en Valladolid a partir de 1485; en Haro en 1465 y 1491; en Segovia en 1483; y en Alba de Tormes a partir de 1458 (Martín Cea, 1991, pp. 124-5; Casado Alonso, 1987, pp. 230, 247, 248; Bennassar, 1983, p. 292, Goicolea Julián, 1999, p. 87; Asenjo, 1986, p. 169; Monsalvo Antón, 1988, pp. 433-5). La incidencia sobre la ganadería estante es evidente y será una constante en la época moderna ${ }^{4}$.

Las hojas de cultivo, un fiable indicador cualitativo, indican simultáneamente una alta densidad poblacional y una relativa escasez de pastos. Sólo necesidades acuciantes justifican la reducción de la libertad económica campesina para permitir un óptimo aprovechamiento pecuario del espacio cerealista. Un procurador de la Puebla de Don Rodrigo (La Mancha) considera que la creación de las hojas se debe a que "se pueden mejor aprovechar los términos e pastarlos con sus ganados" en una situación de "mucha estrechura de términos" (López-Salazar, 1986, pp. 194 y 195). Las hojas inician su desarrollo en el último cuarto del siglo XV. Se documentan o conforman en Zapardiel (Ávila) a mediados del XV, en Ávila en 1487, en Fregeneda (Salamanca) en 1497, en Talavera de la Reina entre 1480-1500, en la zona soriana desde principios del siglo XVI, en la comarca burgalesa en algún momento de la primera mitad del XVI y en este siglo en comarcas segovianas (García Fernández, 1965, p. 703; Suárez Álvarez, 1982, pp. 331-32; Asenjo, 1999, p. 330, n. 285; Díez Sanz, 1995, p. 295; Casado Alonso, 1987, p. 166; García Sanz, 1977, p. 30). En Extremadura, las hojas aparecen entre finales del siglo XV y primera mitad del XVI en Valencia de Alcántara, Plasencia, tierra de Galisteo, Montánchez o Villar de Rena -Medellín- (Clemente Ramos, 2005, p. 62).

Otro elemento a considerar es el desarrollo de la ganadería trashumante en el siglo XV. Sin que tuviera nunca la importancia que se le llegó a conceder, la duplicación de su número a lo largo de este siglo tuvo una incidencia indudable. La cabaña trashumante supera los dos millones y medios de cabezas ovinas en el último cuarto del siglo XV y los tres millones en 1519, para luego descender fuertemente en la segunda mitad del siglo, con menos de dos millones (Le Flem, 1972, p. 38). El equilibrio entre agricultura/ganadería estante y ganadería trashumante se rompe en el reinado de los Reyes Católicos ${ }^{5}$. Las instancias judiciales se llenan de pleitos entre

4 Huetz de Lemps, 1962, p. 248, señala como "Le contingentement des troupeaux apparaît donc comme une disposition fréquente des Ordonnances municipales, partout où la majeure partie du terroir est cultivé, ou de trop faible étendue pour accueillir de nombreuses bêtes"; sobre las limitaciones de la cabaña estante en la época moderna, pp. 247-8.

5 García Sanz, 1998, p. 77, señala como en este momento "se inicia una etapa nueva en la historia de la Mesta: aquella en que es permanente la pugna entre, por una parte, la agricultura integrada con la ganadería estante, que era la base del régimen agrario característico de Castilla durante la época moderna, y, por otra, la ganadería trashumante, completamente ajena a la actividad agricola"; en un sentido similar, Marín Barriguete, 1992, p. 133. 
la Mesta y los concejos. Se registra con claridad la contradicción entre la dedicación pecuaria de las dehesas y la necesidad de tierras de cultivo. En un documento de Enrique IV se precisa que muchos propietarios pertenecientes a las élites "han fecho e fasen dehesas dehesadas la mayor parte de los terminos desa dicha çibdad [Toledo] e su tierra e jurediçion, de que se ha seguido que esa dicha çibdad e los vesinos della e su tierra han padeçido e padesçen mucha hanbre e detrimento asi de pan, porque no hay en que labren los labradores, como de carne, que no ay en que anden e pastan los ganados que son nesçesarios para el proveymiento desa dicha çibdad e su tierra" (Molenat, 1997, p. 509). En Plasencia se registra una situación muy similar. Álvaro de Estúñiga dispuso en 1471 el cultivo del cuarto de las dehesas porque debido a que "les davan por el pasto de las yervas grandes quantías de maravedís, mucho más de lo que por ellas solían dar... no se labravan por pan en esta mi çiudad y su término, en aquella manera que hera nesçesario según la poblaçión de la dicha çiudad y su tierra" (Clemente Ramos y Rodríguez Grajera, 2007, p. 758).

El mundo rural sufre una importante transformación en el siglo XV. En un contexto de fuerte crecimiento demográfico, expansión del espacio agrario, particularmente cerealista, y aumento de la ganadería trashumante, se genera un problema entre el pan, el pasto y, en un claro contexto de retroceso del bosque e incremento de la demanda de productos silvícolas, la madera. Muchas zonas castellanas empiezan a presentar una presión sobre los recursos producto de una ocupación madura, siempre entendida dentro de la estructura de la propiedad y las formas de explotación.

\section{EL MONTE: REDUCCIÓN, ANTROPIZACIÓN Y NUEVAS FORMAS DE EXPLO- TACIÓN}

El crecimiento demográfico y la ampliación del terrazgo agrario, dos variables íntimamente relacionadas, van a tener un impacto extraordinario sobre el monte y la vegetación natural. Se va a producir una aguda antropización del paisaje, una clara disminución del bosque y una mayor demanda, estructural y coyuntural, de productos forestales. El monte empieza a percibirse como un recurso limitado y necesitado de protección. Hasta la segunda mitad del siglo XV, ha sido un bien abundante. No debe extrañarnos ante la limpieza étnica y el vaciamiento demográfico que supuso el proceso expansivo que denominamos reconquista. Algunas fuentes extremeñas presentan con nitidez esta situación. En un documento cauriense de 1553, se alude a un "tienpo ynmemorial" en que no se requería licencia alguna para cortar la madera necesaria y "los montes estaban tan abundosos que llegavan casi a los muros de la çibdad y muy enteros" (Clemente Ramos, 2001, p. 19). En Galisteo, las cercas agrarias se hacían hasta finales del siglo XV con madera de encina y otras quercíneas, pese a la disponibilidad de "otros arvoles que no llevan fruto e como xara, madroño, guadaperales, escobales e azabuches" (Clemente Ramos, 2005, p. 64 y n. 53). Las 
dehesas de Zafra y Zafrilla (Cáceres) eran hacia 1280 "dehesas de coger lande todos aquellos que la quisieren coger asi los de fuera parte como los de la villa et del termino" (Floriano, 1987, doc. 10). Difícilmente podemos imaginarnos una situación más permisiva. Las mismas Cortes de Valladolid de 1351 implícitamente presentan una situación de abundancia donde sólo se intentan corregir unos usos muy agresivos (Clement, 1993, p. 111, n. 103) ${ }^{6}$.

El retroceso del bosque y su consiguiente protección y percepción como bien escaso es algo generalizado desde mediados del siglo $\mathrm{XV}^{7}$. Se registra en la zona cantábrica (García de Cortázar, et al., 1985, vol. II, pp. 82-93; Sánchez Carrera, 1997 , p. 70), la submeseta norte (Casado Alonso, 1987, pp. 37-8, 235; Bennassar, 1983, pp. 36-40; Asenjo, 1999, pp. 346-7, y 1986, pp. 180-2; Clement, 1993, p. 243; Bernal Estévez, 1989, p. 390, n. 102) y sur (Clemente Ramos, 2001 pp. 223; Solano Ruiz, 1978, pp. 339-341; Suárez Álvarez, 1982, pp. 402-5; García Díez, 1987, pp. 73-4) o Andalucía (Borrero, 1982, pp. 106-8; Martín Gutiérrez, 2003, p. 128). Sólo las zonas montañosas y pocas más escaparían a esta situación. Un primer indicador de esta realidad son las ordenanzas de montes. Las ordenanzas contarán con una normativa precisa sobre la protección y explotación del bosque con la clara finalidad de garantizar la sostenibilidad de un recurso limitado (Luchía, 2014). Las normativas proteccionistas se desarrollan con fuerza en Extremadura a finales del siglo XV (Valencia de Alcántara, 1489; Cáceres, 1494; Trujillo, 1499; Coria, 1502), dinámica que seguirá en el siglo siguiente (Clemente Ramos, 2001, p. 25).

La problemática del bosque trasciende el ámbito local desde finales del siglo XV. Los Reyes Católicos promulgan las primeras normativas proteccionistas de carácter general. Carlos I y Felipe II continúan esta política. Las Cortes, igualmente, presentan un interés creciente sobre la materia (1538, 1573 ó 1576). Las de Madrid de 1573 señalan, quizás de modo un tanto exagerado, que en Andalucía y el reino de Toledo "ya no se halla ni puede quemar sino sarmientos y paja". En las de 1576, se solicita que se priorice la aprobación de las ordenanzas de montes pendientes (Bauer, 1980, pp. 54-5 y 63).

La problemática del bosque parece intensificarse en el siglo XVI. En una provisión de 1567 que autoriza la repoblación forestal en Burgos se precisa que "la tierra

6 "se destruyen de cada dia de mala manera los montes, senalada miente los pinares e enzinares, porque derriban çinco o seyx pinnos por tirar dende tres o quatro rrayeros de tea que non valen tres dineros, et que en los enzinares, por un palo muy sotil que ayan menester que cortan un enzina por pie" (cit. Clément, "Frontière", 111, n. 103).

7 Una sensibilidad distinta se detecta ya en la segunda mitad del XIV, aunque de forma un tanto tímida e incipiente, en zonas de antigua ocupación. El monasterio de San Bartolomé de Navas (Asturias) muestra un interés en 1362-6 en la conservación del bosque y prohíbe la tala de árboles. El cabildo de la iglesia de Santa María de Valladolid exige en 1338 a los arrendatarios de las huertas del Pisuerga la plantación de árboles para la construcción y otros usos (Torrente Fernández, 1982, pp. 97-8; Rucquoi, 1997, p. 297). 
en la mayor parte destos reynos esta yerma y rasa sin arboles algunos y que la leña y madera a benido a faltar de manera que ya en muchas partes no se puede vivir" (Coronas Vida, 2004, p. 159). En Asturias, La Junta General del Principado (1595) señala que en los montes concejiles y comunes se han realizado "muchos cortes de enzinas y robres y otras frutas, de cuia causa vienen en disminucion y no se conservan" (Barreiro, 1992, 248). En Alcaraz, a raíz de la provisión de Felipe II sobre los montes, una investigación concluye que las encinas son "pocas segund los que solia aver". En este lugar y en Almansa, la repoblación parece una vía ineludible (Pereda Hernández, 1988, pp. 90-1). La tierra de Soria es un ejemplo paradigmático. En 1518, fue necesario elaborar unas ordenanzas de montes. A mediados de siglo, Juan Ochoa de Urquizu se quejó ante el Consejo Real de que los montes, antes muy poblados, "se habían alargado mucho por las cortas y talas", por lo que el radio del abastecimiento de leña había aumentado de tres a ocho-diez leguas. En 1582, los regidores constatan el deterioro del monte de Valonsadero y prohíben "cortar o rozar cualquier genero de arbol, ya sea seco o verde". Ese mismo año, Soria alarga la veda por dos años más de los habituales en el monte de la Aldehuela de Periáñez, propio de la ciudad. En 1593, la Junta de la Tierra precisa que "en muy pocas aldeas había montes de bellota de los que se pudiera sacar algún aprovechamiento". Algunas aldeas intentarán aumentar la protección de sus montes para facilitar su recuperación. Sin embargo, el procurador de causas de la ciudad acusa a los vecinos de cuarenta y seis aldeas en 1600 de "haber cortado mucha cantidad de pies y ramas de roble y carrasca". Los acusados arguyen que se trata de comunes y propios de las aldeas y que pueden cortar "pie y por rama" sin autorización de la ciudad, lo que finalmente ratificó la justicia (Díez Sanz, 1995, pp. 119-121 y 124). Sin entrar en la incidencia que las demandas de la marina real va a tener sobre el bosque, lo que será una constante para el periodo moderno, es significativo que se tendrá que considerar la replantación como una política necesaria e ineludible (Rey Castelao, 1995, pp. 1678; Rivero Rodríguez, 2005, pp. 355-6).

El retroceso del bosque va a producir el aumento de los precios y el desarrollo de un comercio intercomarcal (mal conocido) e interregional de los productos forestales. Los datos sobre precios son escasos pero significativos. En Medellín, el precio de las barcas del Guadiana, según las estimaciones más moderadas de diversos testigos, se duplica o triplica en el tercer cuarto del siglo XV (Clemente Ramos, 2016, pp. 138-140). En Soria, la carretada de leña pasa de 1,5 reales a principios del XVI a seis a mediados de siglo, aunque su precio legal estaba limitado a tres, por lo que los pobres "llegaban a gastar mas en leña que en comer" (Díez Sanz, 1995, 117). En 1595, la situación del bosque como hemos visto parece complicada en Asturias. No sorprende que en las décadas siguientes el precio de la madera suba mucho más deprisa que el de la paja o el cereal sin que se registre un claro aumento demográfico (Barreiro, 1992, pp. 248-9). 
La nueva situación del bosque y su percepción como un recurso limitado va a generar un nueva silvicultura que intenta desarrollar un modelo de explotación sostenible. La tala de un árbol o la saca de madera van a requerir una previa autorización. En el bajo Miño, los foros privados prohíben la tala "por pee" a la vez que limitan o reservan al propietario el aprovechamiento de la madera (Sánchez Carrera, 1997, p. 70). Las ordenanzas de Soria de 1497 también prohíben la tala de hayas, encinas, robles o pinos (Asenjo, 1999, pp.346-7). Diversas ordenanzas de la vertiente meridional del Duero siguen esta línea proteccionista desde finales del siglo XV al XVI -Cuellar, 1493 y 1546-8, Riaza, 1572; Coca, 1623-(Clement, 1993, p. 243). La escasez de madera en Talavera obligará a la redacción de las ordenanzas de 1508 (Suárez Álvarez, 1982, pp. 402-5). Las ordenanzas de Alcaraz de 1521 intentan poner coto a la tala abusiva y proteger un recurso en claro retroceso. La tala por el pie queda reservada a arbustos como enebros, madroños y aladiernos, considerados de menor interés (García Díaz, 1987, pp. 85-90). Alcántara sostendrá en un conflicto con la Mesta en 1523 que en su término "no avia monte que se avastase para haser puentes sy no dexasen horca e aljuma" (Clemente Ramos, 2000, p. 327). En definitiva, el abastecimiento de madera se intentará realizar siempre que sea posible con el adecuado desmochado (poda) del arbolado y no con la tala de ejemplares.

El desarrollo de nuevas estrategias y su generalización, al menos en contextos de escasez, está relacionado con la necesaria sostenibilidad forestal. La repoblación empieza a aparecer como un política ineludible. A finales de la Edad Media está ampliamente asentada incluso en zonas cantábricas. Bilbao la desarrolla desde 1495. También lo hace el monasterio de Meira en tierras del Eo y en la ribera monfortina del Sil (García de Cortázar, 1985, II, p. 8; Mariño Veirás, 1983, 318-9). En Burgos, la reina Juana I dispone la plantación de pinos, sauces y álamos, lo que repetirá su hijo Carlos I y se continuará especialmente en el último tercio del siglo XVI (Casado Alonso, 1987, pp. 37-8 y 235; Coronas Vida, 2004, pp. 157-183). El caso de Valladolid está bien estudiado. Desde 1495 se plantan pinos y encinas. En el siglo XVI el panorama forestal cambia completamente. Se desarrollan grandes pinares, sobre todo al sur de la ciudad, convirtiéndose en la asociación vegetal dominante (Bennassar, 1983, pp. 36-40). En Andalucía también se práctica la repoblación. En Sevilla, durante el siglo XVI se desarrolla en "montes y pinares" y en las riberas, con "salzes y alamos" (Borrero, 1983, p. 97). Unas ordenanzas de 1538 de Úbeda recomiendan la plantación de álamos y pinos por cada árbol que se tale (Parejo, 1988, p. 106).

El uso creciente del matorral y de los árboles y arbustos de menor interés económico intentará aliviar la presión sobre el bosque. El ejemplo de Galisteo, que estudié hace algunos años, es claro en este sentido. Tradicionalmente se llevaba leña verde y seca a Plasencia y se introducía de esta jurisdicción madera para la construcción. Esto indica una relativa escasez de monte alto. Los oficiales intentan promover una explotación más racional de los recursos mediante el aprovechamiento de los bos- 
ques "espesos e renesidos", que de este modo se preparaban para un aprovechamiento pecuario, y la prohibición de cortar leña verde "para quemar ni haser arados, ni para cerrar sus huertos, ni para dar de comer a las reses flacas, ni para los bueyes de arada, ni para otra cosa ninguna". Consideran fundamental utilizar "otros arvoles que no llevan fruto [bellota] e como xara, madroño, guadaperales, escobales e azabuches" (Clemente Ramos, 2005, pp. 62-4).

La información sobre la explotación del matorral y de arbustos, o de árboles que ofrecen un menor interés, se acumula desde finales del siglo XV. En Cáceres (a. 1494), se ordena la sustitución de la leña de alcornoque por la de "escoba, e xara, e lantisca e otras lennas". En Coria (Cáceres), los hornos de pan deberán utilizar "xara e madroño e hornija" (Clemente Ramos, 2001, p. 26). En Becerril de Campos, con una limitada riqueza forestal, se utilizan como combustible los cardos, las escobas o las zarzas (Oliva Herrer, 2002, pp. 129-130). En Alcaraz, en las ordenanzas de la caballería de la sierra de 1521, bastantes restrictivas, se permite cortar por el pie todo el año enebros, madroños y aladiernos y las ramas de sauces, fresnos y coscojas (García Díaz, 1987, pp. 85-90). La evolución de las condiciones de arrendamiento de las dehesas del linaje toledano de los Niño es muy indicativa. En la primera mitad del XV, los arrendadores podían hacer un uso prácticamente ilimitado de la retama. A partir de 1485, se prohíbe o limita su aprovechamiento de forma precisa y a su vez se ponen condiciones mucho más restrictivas para la explotación de los productos forestales (Franco Silva, 2005, pp. 157-166). Las Relaciones Topográficas de Felipe II registran en la provincia de Madrid el aprovechamiento para leña, además de las quercíneas, de la jara, el romero, la retama, la aliaga, el tomillo, el labiérnago, la cornicabra o el enebro (Bauer, 1980, pp. 115-6).

El comercio de los productos forestales y especialmente de la madera pasará a estar crecientemente controlado por los concejos. En general, se desarrolla una oposición entre zonas de montaña y llanura, con pocos bosques o sin monte alto, que sin duda fijaría con precisión una investigación sistemática. La montaña extremeña presenta un perfil paisajístico preciso caracterizado por una mayor riqueza forestal. Plasencia alegará frente a la provisión real de 1567 que cuenta con bosques abundantes. Se localizan, de modo preferente, en las zonas de montaña (Clemente Ramos, 2015, pp. 544-549; Clemente Ramos \& Rodríguez Grajera, 2007, pp. 739741). No es raro que el comercio de la madera afecte a otros productos. Talavera permite a principios del XVI la saca de pan en momento de aguda penuria cerealista a Mombeltrán o Arenas de San Pedro, puntos habituales de abastecimiento de madera (Suárez Álvarez, 1982, 402-5). Alcaraz presenta la situación contraria: obliga a los forasteros a la introducción de pan para la saca de madera desde 1462 (García Díaz, 1987, pp. 73-4).

Plasencia, en Extremadura, es un ejemplo del comercio intercomarcal de madera. La ciudad y su término es excedentaria con sus pinares, que jalonan el río Tiétar, 
y los castañares serranos. Se exporta madera hacia Galisteo, Alconétar, Alcántara o Trujillo. El control de este comercio se hace cada vez más riguroso y la ciudad se hace confirmar por Carlos I, de modo sólo preventivo, su prohibición (Clemente Ramos, 1999, pp. 6-7). Otras zonas presentan un modelo diferente, con montes que sostienen un comercio interregional. Las encomiendas santiaguistas del reino de Murcia y la sierra de Segura, predominantemente montañosas, suministran madera a la región y a Andalucía, sobre todo a partir de 1480 (Rodríguez Llopis, 1984, pp. 238-43). En la Sierra Pinariega, al noroeste de Soria, lugares pertenecientes a distintas jurisdicciones forman la Hermandad de Pinares. La madera de la zona llega a Burgos, Zamora, Palencia, León y Astorga, Valladolid o Medina del Campo a finales del siglo XV (Asenjo, 1999, p. 347). Córdoba puede ser un buen ejemplo de la dificultad que presentaba el abastecimiento de madera. Recibía madera de pino fundamentalmente de la Sierra de Segura. La madera local se obtenía en zonas montañosas (Pedroches, Sierra de Hornachuelos) o en unos montes concejiles muy protegidos y sujetos a un control riguroso. Con todo, se debió realizar una ordenanzas de carpinteros para responder "a la poca madera que hay en esta ciudad" (Córdoba de la Llave, 1990, p.276; en general pp. 272-7).

Las nuevas formas de explotación maderera han introducido cambios en el bosque. En casos específicos, va perdiendo su carácter estrictamente natural. Sin duda, es un tema que merecería estudios detenidos. Hasta el siglo XV, la madera utilizada ha debido provenir sobre todo de especies climácicas o de ribera y su abastecimiento ha debido realizarse preferentemente dentro de cada jurisdicción o en un radio reducido. Su comercio ha generado, por ello, una escasa información. A lo largo del siglo XV, cada vez es más frecuente un déficit total o más habitualmente parcial de recursos forestales, especialmente de monte alto. Este recurso sólo esta disponible en situaciones de explotación equilibrada, puesto que la sobrexplotación reduce el porte del arbolado. Por otro lado, un suministro abundante y regular de madera exige la explotación de especies de rápido crecimiento como el pino. La vegetación de ribera ha sufrido un retroceso muy importante por la calidad de los espacios que ocupa y su limitado interés pecuario (Clemente Ramos, 2001, 36-42; Clemente Ramos y Martín Gutiérrez, 2018, 43-50). En los grandes macizos forestales vemos la explotación de pinares, de origen probablemente espontáneo. En otros casos, como los pinares que jalonan el Tiétar en la tierra placentina, es probable un origen o un componente antrópico, algo documentado en el caso vallisoletano para el siglo XVI.

\section{CAZA Y FAUNA: LA LUCHA CONTRA EL LOBO}

La antropización del paisaje y el retroceso de la vegetación natural incidieron fuertemente sobre la fauna salvaje. La lucha contra el lobo es un claro indicador de los desequilibrios generados. Otras especies como el oso y el cebro/encebro (asno 
salvaje), se han visto condicionadas ya desde el siglo XIII por las transformaciones ambientales. El oso es un animal muy sensible a la actuación humana (Clevenger y Purroy, 1991, p. 90). La Extremadura histórica contaba a finales del siglo XI, como hemos indicado, con una abundante población (Clément, 1993, p. 92). A mediados del siglo XIV, ha ido abandonando la llanura y se ha convertido en un animal de montaña, lo que se ha cartografiado con precisión para la Extremadura actual. Igualmente, estaba ausente en la depresión del Guadalquivir (Clemente Ramos, 2015, p. 566, mapa 1; López Ontiveros et al., 1988, p. 294). En el siglo XVI, el espacio del oso se va reduciendo lentamente: no se documenta en Madrid, Cuenca o el reino de Granada. Esta dinámica se mantendrá a lo largo del periodo moderno. El oso pierde presencia en la literatura cinegética (Nores y Naves, 1993, pp. 4-5). Otro ejemplo de la creciente antropización del paisaje y de su incidencia sobre los grandes herbívoros es el cebro (iequus hydrauntinus?). Ampliamente extendido en el siglo XII en el occidente y centro peninsular, desde el siglo XIV sólo se documentan en la zona de Murcia/Albacete. Las relaciones Topográficas de Felipe II certifican su extinción (Nores Quesada y Liesau, 1992, pp. 65 y 68-9). Estos dos ejemplos nos muestran la temprana incidencia de la creciente antropización del espacio antes de su definitiva agrarización sobre las especies más sensibles y con menos capacidad de adaptación.

El problema del lobo se manifiesta tempranamente, en el siglo XIII y primera mitad del XIV, en las zonas más humanizadas como Galicia (Sobrado, 2003, pp. 107-8). En la segunda mitad de este siglo se registra el pago de recompensas por capturas en Murcia (Menjot, 1980, p. 264). La lucha contra el lobo se va a generalizar a finales del siglo XV en Extremadura, Andalucía, Murcia o el espacio castellano-manchego. También en el norte. En todo caso, parece un problema percibido dentro del ámbito local. En el siglo XVI, las Cortes le otorgan ya una dimensión interregional. No sorprende, por la íntima relación entre paisaje vegetal y fauna salvaje, que esta preocupación presente a grandes rasgos una cronología coincidente con la evolución del bosque y su protección.

Las grandes transformaciones económicas bajomedievales alteraron significativamente el hábitat de la fauna salvaje y, especialmente, de los grandes herbívoros, básicos en la alimentación del lobo. La caza menor se vio menos afectada. En todo caso, la proliferación de ordenanzas de caza está indicando el retroceso de todas las poblaciones animales. Las cortes de Madrid de 1435 ya muestran la incidencia que sobre liebres y perdices tienen las técnicas más indiscriminadas (Torquemada, 1997, p. 99). La novedad de las nuevas medidas de protección no deja lugar a dudas. En 1492, el común de los vecinos de Cáceres se posiciona contra las nuevas normas que rompen con lo que "antiguamente solian usar" (Clemente Ramos, 2001, p. 45). En Extremadura, se perfilan unas pautas sobre la caza, particularmente la menor, que creemos que en líneas generales puede extenderse a otros muchos territorios. Se prohíben las técnicas más mortíferas e indiscriminadas, se establecen vedas en 
la época de cría y se reservan los productos cinegéticos para el consumo local (Clemente Ramos, 2001, p. 45: Ladero Quesada, 1980, pp. 201 y 216-7). Todo indica, aunque faltan estudios consistentes y rigurosos, que estas medidas permitieron mantener las poblaciones de caza menor. Muchas de estas especies se adaptan mejor a una mayor presencia de la agricultura (perdiz, liebre, conejo, etc.). Esto explicaría situaciones puntuales de abundancia, que en todo caso parecen excepcionales, en el siglo XVI (Clemente Ramos, 2001, p. 48; Goicolea Julián, 1999, p. 91, n. 292; Del Pino García, 1996, p. 105).

Los profundos cambios ambientales que se desarrollan a lo largo del siglo XV incidieron muy fuertemente sobre los grandes herbívoros, más dependientes del monte y las densas manchas forestales. Sobre el particular, es muy significativo el interés preferente de las ordenanzas en la caza menor (Ladero, 1980, pp. 201 y 216-7). En un tema particularmente mal estudiado, donde hay que moverse con informaciones de carácter cualitativo no exentas de ambigüedad, quizás sea muy significativa la proliferación de sitios reales y cotos señoriales. Los sitios reales eran, al igual que las grandes fincas cinegéticas actuales, cotos y criaderos de caza. En 1579, aldeas próximas al monte del Pardo entregaron un memorial sobre los daños producidos por los animales salvajes para su compensación (Rivero Rodríguez, 2005, p. 358). En 1494, el monte del cazadero real del Lomo del Grullo, junto a Doñana, quedó vetado al ganado y se prohibió que nadie cazara jabalíes o ciervos entre otras especies cinegéticas en una legua alrededor del palacio. En 1495, este espacio se repobló con ciervos. En 1534, se prohibió la entrada de ganado y la bellota se reservó para las especies cinegéticas, especialmente el jabalí (López Viera, 2002, pp. 403-4).

La nobleza también estableció cotos de caza. En Haro, el condestable Iñigo Fernández de Velasco acordó en 1525 con el concejo el vedamiento de la caza y la pesca en el soto situado a orillas del río Oja a cambio de la cesión de sus derechos sobre las alcabalas de Naharruri (Goicolea, 1999, p. 91, n. 300). En 1504, la dehesa del Bodegón (Puebla de Alcocer, Badajoz), propiedad del duque de Béjar, era "un coto con mucha caza y una casa de recreación en las riberas de Guadiana" (Torres y Tapia, 1999, vol. II, p. 614). Las relaciones topográficas de Felipe II nos ofrecen algunos ejemplos muy claros. En Taragudo (Guadalajara) ) las "alimañas salvajinas" sólo se crían en los vedados del Duque del Infantado porque el resto del término "es tierra rasa y no hay montes". En San Martín de Valdepusa (Toledo) hay un contraste entre la dehesa señorial de Pedro de Ribera, en la que se crían "muchas perdices, conejos y liebres y gamos y venados algunos, y puercos jabalies" y el resto del término, labrantíos que sólo permiten la "caza menuda" (Arroyo Llera, 1991, p. 190). En 1543, en un pleito contra el conde de Medellín se detalla su intento de monopolizar la caza del término, vedándola a los vecinos y la oligarquía local, y las violencias cometidas contra personas y animales (perros) ${ }^{8}$. Todos estos elementos

8 A. G. Simancas, Consejo Real, leg. 230, exp. 2-1, preg. 11, fols. 11r. 
indican un claro retroceso de la caza y particularmente de los grandes herbívoros. Los intentos de protección y el desarrollo de zonas reservadas (sitios reales o cotos señoriales) tienen una clara significación. En Castilla, sin embargo, no se llegará al monopolio de la caza por las élites como en otros entornos europeos (Montanari, 1984, pp. 180-3; Almond, 2003, pp. 33, 93-4 y 141).

Las profundas transformaciones ecológicas bajomedievales van a cambiar la relación entre el hombre y el lobo. Situado en la cúspide de la cadena trófica, la presencia del lobo va adquiriendo un perfil conflictivo en Europa a lo largo del periodo medieval. Esta situación está estrictamente relacionada con la antropización del paisaje y el subsiguiente retroceso de los grandes herbívoros. Los espacios del hombre y el lobo chocan cuando este animal, privado de sus fuentes naturales de alimentación, se convierte en una importante amenaza para la ganadería.

Los problemas planteados por el lobo tienen un evidente componente trófico. Varios indicadores apuntan en este sentido. Debemos considerar, en primer lugar, el papel central que tienen los grandes herbívoros en su alimentación (Grande del Brío, 1984, pp. 44-5; Gil Cubillo, 1991, pp. 149-50; Kemp, 1990, p. 79). Ante su escasez, la ganadería cumple un papel sustitutivo, al menos estacionalmente. La información sobre el particular es clara y precisa. Las ordenanzas de Chinchilla indican que "façian muchos daños e muertes [los lobos] en los ganados mayores, e menores, e bestiales". En Jerez o Lorca, los ganaderos pagan las recompensas por las capturas (Torquemada, 1997, pp. 76-7; Carmona, 1998, p. 413; Jiménez Alcázar, 1997, pp. 113-4). En Extremadura, los datos son relativamente abundantes. Las cuentas municipales de Cáceres de 1519 registran veinticinco más un número indeterminado de carneros víctimas del lobo. En 1508, la aldea de Garciaz (Trujillo) solicita quemar una mancha con mucho monte porque "reçiben mucho daño en sus ganados de lobos". Las ordenanzas de Valencia de Alcántara (a. 1486) también aluden a las reses muertas por estos animales. El lobo también ataca al ganado mayor como hacen constar las ordenanzas de Villalba (a. 1549) y Solana de Barros (a. 1554). Los mastines se convierten en imprescindibles. Los monjes de Guadalupe, ante la eventualidad de mantener atados a sus perros por haber atacado a ganado doméstico de Rena y Villar (Medellín), argumentan que en ese caso "les comerian los lobos el dicho ganado", prioritariamente bovino. En Haro, el uso de mastines parece tener la misma finalidad (Clemente Ramos, 2000, p. 370 y n. 163, y 2001, pp. 48-9; A. Mon. Guadalupe, leg. 131, doc. 56; Goicolea Julián, 1999, p. 89).

La estacionalidad de las capturas es otro claro indicador de la naturaleza del conflicto9. Las capturas dibujan a lo largo del año una línea invertida respecto de la disponibilidad de alimentos. De este modo, registran un pico en invierno y son

9 Cherubini, 1996, p. 203, señalaba en un trabajo clásico que "i lupi adulti risultino presi nel periodo invernale, quando evidentemente essi si facevano più imprudenti a causa della fame, mentre la tarda primavera e l'estate erano la stagione della cattura dei cuccioli nelle boscaglie"; en general, págs. 203-6 
más abundantes en el primer semestre del año, mientras que escasean en el verano y el otoño. En Extremadura, con datos de varios concejos para finales del siglo XV y primera mitad del XVI, se alcanza un máximo en enero-febrero y un segundo en mayo. Las camadas de lobeznos se capturan, por razones obvias, en abril-mayo. En Lugo, para el periodo moderno, el máximo de capturas también se sitúa en enerofebrero (Clemente Ramos, 2001, p. 49; Sobrado, 20003, p. 111) ${ }^{10}$.

Pese a su gran capacidad de adaptación, el lobo acusa los cambios y aunque puede considerarse omnipresente, algo facilitado por su movilidad, prolifera en las zonas con menos transformación ambiental ${ }^{11}$. Sólo podemos esbozar algunas líneas, ante la inexistencia de estudios sobre la materia. Aunque presente en el llano, se asienta preferentemente en las zonas de montaña o donde la existencia de abundantes matas permite su proliferación. Las cortes de Valladolid de 1548 y las de Madrid de 1551 señalan esa vinculación del lobo y de otras alimañas con las montañas de Galicia y Extremadura. En esta última región, tiene una presencia especial en la Sierra de Guadalupe, Sierra Morena y zonas colindantes de Portugal (Ladero, 1980, p. 202). Las montañas lucenses albergan una abundante población en el siglo XVIII (Sobrado, 2003, pp. 120-1). En Extremadura se ha podido estudiar su reparto en algunos términos concejiles. En la tierra de Cáceres, parecen abundantes en la Sierra de San Pedro y en las estribaciones montañosas que separan la villa de Sierra de Fuentes. En Mérida, proliferan en las proximidades de la Sierra de San Pedro y Montánchez al norte y junto a la Sierra de San Serván al sur (Clemente Ramos, 2001, p. 51).

Este relación entre poblaciones de lobos y zonas de monte y matorral nos indica la relación estrecha entre paisaje vegetal y fauna. El hombre medieval y moderno era consciente de la dificultad de eliminar al lobo y una de sus estrategias será la eliminación de su hábitat. La dehesa de Cíjara (Montes de Toledo) era "muy lobosa por las grandes malesa", por lo que Toledo autorizó la roturación de espacios agrarios (Molenat, 1997, p. 266, n. 112). La Sierra de Baza se aprovechaba poco en el primer cuarto del siglo XVI porque "en la espesura dellos se crian muchos lobos e otros anymales" (Tristán García, 2000, p. 243). Del mismo modo, en Trujillo se piden desbroces para controlar su población -"quemar un pedaço de la dehesa, que esta muy montosa y reçiben mucho daño en sus ganados de lobos"- (Sánchez Rubio, Trujillo, pp. 201 y 205-6, notas 200 y 202). La dehesa de Castilrubio (Medellín), con una gran presencia de montes bravos y abundante vegetación era un lugar perfecto para la proliferación del lobo y los grandes herbívoros al menos hasta 1450 -"muchos javalies e venados e caça e muchos lobos"- (Clemente Ramos, 2019 , p. 137, n. 12).

10 Igualmente, las capturas de lobos en Castellón entre 1566-1624 se realizan también de modo muy acusado entre enero y julio (Rosas, 2009, p. 7).

11 Sobre la proliferación del lobo en entornos con bosque, ganadería ovina y poca población en la Francia del siglo XVIII, cf. Molinier y Molinier-Meyer, 1981, pp. 231-4. 
Esta necesidad de alterar los ecosistemas vegetales es un indicador de la dificultad de eliminar o reducir la población de lobos. Los éxitos fueron escasos y las capturas no supusieron un claro retroceso salvo en espacios y zonas muy puntuales o insulares (Ortalli, 1997, pp. 79-82; Almond, 2003, p. 71, Grau, Puig y RuizOlmo 1990, p. 221; Rosas, 2009, p. 8). No estamos en condiciones de establecer la evolución de sus poblaciones, pero todo hace indicar que de modo general se mantuvieron en la baja Edad Media y el periodo moderno. Cuando vemos grandes zonas, los cambios no parecen importantes. En Galicia siguen siendo abundantes en el periodo moderno y suponen un claro problema (Sobrado, 2003, pp. 120-1). En la baja Extremadura, a través del interrogatorio de la Real Audiencia de 1791 se registra la presencia del lobo en el 83 \% de los municipios (Grajera, 1996, pp. 30-1).

El problema del lobo, endémico entre la baja Edad Media y el siglo XIX, hunde sus raíces en las transformaciones y desequilibrios ambientales de los siglos XV-XVI. Los espacios del hombre y el lobo chocan sin que, debido a la adaptabilidad de este animal, pueda eliminarse su presencia. El mundo rural ha terminado interiorizando una amenaza que aún se manifiesta de forma clara frente a los actuales intentos conservacionistas.

\section{CONCLUSIONES}

La fase de crecimiento que se extiende entre comienzos del siglo XV y gran parte del siglo XVI, generó un aumento considerable de la población. Las zonas y territorios con menor ocupación, situadas al sur del Sistema Central y conquistadas tardíamente, alcanzan ahora un nivel demográfico importante considerando las estructuras de la propiedad y las formas de explotación. Esto produjo, dentro de un modelo de crecimiento de carácter extensivo, una proliferación de roturaciones ante las crecientes demandas alimenticias. El paisaje se agrariza y el monte y la vegetación natural sufren un claro retroceso. El bosque se ha visto hasta el siglo XV, sobre todo en las zonas meridionales, como un recurso abundante. Desde mediados de este siglo, las ordenanzas de monte empiezan a manifestar una clara preocupación y la existencia de una nueva realidad definida por la escasez relativa. Las nuevas medidas conservacionistas se convierten en imprescindibles. En el siglo XVI, la legislación real y las Cortes se harán eco de la situación. Asistimos al desarrollo de repoblaciones forestales. A partir de ahora, el trigo y la madera aparecen como dos productos imprescindibles pero con requerimientos opuestos. La comercialización de la madera estará crecientemente controlada.

El importante retroceso del bosque y la vegetación natural ha tenido una fuerte incidencia sobre la caza y la fauna salvaje. Antes del siglo XV, sólo las especies con una menor adaptabilidad, como el oso o el cebro/encebro, acusan la creciente trans- 
formación del espacio. En los siglos XV y XVI, la intensa agrarización y antropización produjo efectos generales sobre la fauna. Las ordenanzas de caza y el desarrollo de cotos reales y señoriales son un claro indicador de esta realidad. Los grandes herbívoros se han visto especialmente afectados. Privado de su principal fuente de alimentación, el lobo se convierte en una amenaza para la ganadería. Las recompensas por sus capturas se generalizan. Animal con gran capacidad de adaptación, seguirá siendo omnipresente, especialmente en las zonas menos transformadas, y mantendrá sus poblaciones salvo contadas excepciones durante siglos. A pequeña escala, la eliminación de las manchas densas que le sirven de refugio parece la forma más sencilla de limitar su proliferación.

Hacia 1500, agricultura, monte y fauna aparecen como tres elementos clara e íntimamente relacionados. El crecimiento de la población y la antropización y agrarización del espacio han producido un claro retroceso del bosque y la caza, que pasarán a estar creciente y sistemáticamente protegidos, y el conflicto con el lobo, que ve reducido drásticamente su hábitat y su base alimenticia.

\section{REFERENCIAS BIBLIOGRÁFICAS}

Almond, R. (2003). Medieval hunting. Stroud (Gloucestershire): Sutton Publishing. Arroyo Llera, F. (1991). Caza y fauna en Castilla la Nueva en el siglo XVI según las

Relaciones Topográficas de Felipe II. En Actas del VI Coloquio de Geografia Rural (pp. 183-196). Madrid: UAM.

Asenjo González, Ma (1986). Segovia. La ciudad y su tierra a fines del medievo. Segovia: Diputación de Segovia.

Asenjo GonZÁlez, Ma (1999). Espacio y sociedad en la Soria medieval (siglos XIII$X V)$. Soria: Diputación de Soria.

BARReiro Mallón, B. (1992). Masa arbórea y su producto en Asturias durante la Edad Moderna. En V. Cabero, J. M. Llorente Pinto, J. I. Plaza Gutiérrez y C. Pol Ménde (Eds.), El medio rural español. Cultura, paisaje y naturaleza (pp. 240-252). Salamanca: Universidad de Salamanca.

BAUER MANDERSCHEID, E. (1980). Los montes de España en la historia. Madrid: Ministerio de Agricultura.

Bernal EstéVez, A. (1989). El concejo de Ciudad Rodrigo y su tierra durante el siglo $X V$. Salamanca: Diputación de Salamanca.

Bennassar, B. (1983). Valladolid en el Siglo de Oro. Una ciudad de Castilla y su entorno agrario en el siglo XVI. Valladolid: Ayuntamiento de Valladolid.

Blanco Carrasco, J. P. (1999). Demografía, familia y sociedad en la Extremadura moderna, (1500-1860). Cáceres: Universidad de Extremadura.

BORRERO FERNÁNDEZ, M. (1983). El mundo rural sevillano en el siglo XV: Aljarafe y Ribera. Sevilla: Universidad de Sevilla. 
CARMOna Ruiz, Ma A. (1998). La ganadería en el Reino de Sevilla durante la Baja Edad Media. Sevilla : Diputación de Sevilla.

Casado Alonso, H. (1987). Señores, mercaderes y campesinos. La comarca de Burgos a fines de la Edad Media. Valladolid: Junta de Castilla y León.

Cherubini, G. (1996). Lupo e mondo rurale. En G. Cherubini, LItalia rurale del basso Medioevo. Roma-Bari: Laterza

ClÉment, V. (1993). Frontière, reconquête et mutation des paysages végetaux entre Duero et système central du XIe au milieu du XVe siècle. Melanges de la Casa de Velázquez, XXIX/1, 87-126. https://doi.org/10.3406/casa.1993.2640

Clemente Ramos, J. (2003). Los deslindes: una fuente para el estudio de la vegetación natural. En J. Pérez-Embid (Ed.), La Andalucía Medieval. Actas de la I Jornadas sobre Historia rural y medio ambiente (pp. 129-142). Huelva: Universidad de Huelva.

Clemente Ramos, J. (2005). Desarrollo agrario y explotación forestal en la tierra de Galisteo a finales de la Edad Media. En Clemente Ramos y J. L. de la Montaña Conchiña, II Jornadas de Historia Medieval de Extremadura. Ponencias y comunicaciones (pp. 57-74). Mérida: Editora Regional de Extremadura.

Clemente Ramos, J. (2015). La montaña extremeña (ss. XV-XVI). Paisaje y economía. Revista de Estudios Extremeños, (71/1), 539-568.

Clemente Ramos, J. (2005). La organización del terrazgo agropecuario en Extremadura (siglos XV-XVI). En la España Medieval, (28), 49-80.

Clemente Ramos, J. (2016). Barcas y barcaje en Medellín (c. 1449-c. 1511). Riparia, (2), 133-152. https://doi.org/10.25267/RIPARIA.2016.v2.07

Clemente Ramos, J. (2019). Una dehesa por dentro: Castilrubio (1290-c. 1545). Paisaje, explotación y usurpación de términos. Espacio, Tiempo y Forma. III. Historia Medieval, (32) , 133-160. https://doi.org/10.5944/etfiii.32.2019.22235

Clemente Ramos, J. y Martín Gutiérrez, E. (2018). I paesaggi d'acqua e le loro utilizzazioni nella Spagna sudoccidentale tra il tredicesimo e il sedicesimo secolo. En Gestione dell'acqua in europa (XII-XVIII Secc.) - WaterManagement in Europe (12th-18th centuries): selezione di ricerche - Selection of essays (pp. 41-61). Firenze: Firenze University Press.

Clemente Ramos, J. y Rodríguez Grajera, A. (2007). Plasencia y su tierra en el tránsito de la Edad Media a la Moderna. Un estudio de sus ordenanzas (14691593). Revista de Estudios Extremeños, LXIII/2, 725-789.

Clevenger, A. P. y Purroy, F. J. (1991). Ecología del oso pardo en España, Madrid: CSIC.

Collantes de Terán, A. (1984). Sevilla en la baja edad media. La ciudad y sus hombres. Sevilla: Ayuntamiento de Sevilla.

Córdoba de la Llave, R. (1990). La industria medieval de Córdoba. Córdoba: Universidad de Córdoba. 
CORONAS VIDA, Luis Javier (2004). Montes, comunales y repoblación forestal en la jurisdicción de Burgos durante el siglo XVI. Boletín de la Institución Fernán González, 228, 153-192.

DíEz SANZ, E. (1995). La tierra de Soria. Un universo campesino en la Castilla oriental del siglo XVI. Madrid: Siglo XXI.

FERnÁNDEZ Petrement, L. (1988): Licencias de Rotura y cerramientos de dehesas en el Campo de Montiel (s. XVI). En I Congreso de Historia de Castilla-La Mancha. 7. Conflictos sociales y evolución económica en la Edad Moderna (pp. 97-105). Toledo: Junta de Comunidades de Castilla-La Mancha.

Floriano, A. C. (1987). Documentación histórica del Archivo Municipal de Cáceres (1229-1471). Cáceres: Diputación de Cáceres.

Franco Silva, A. (2005). Los Niño. Un linaje de la oligarquía municipal de Toledo en el siglo XV. En A. Franco Silva, Estudios sobre la nobleza y el régimen señorial en el reino de Castilla (pp. 127-213): Cádiz: Universidad de Cádiz.

García de Cortázar, J. A.; Arízaba Bolumburu, B; Luz Rodriguez, Ma L.; y Del Val Valdivieso, I. (1985): Bizcaya en la Edad Media. Evolución demográfica, económica, social y política de la comunidad vizcaína medieval, 3 vols. San Sebastián: Haranburu Editor.

GarCía DíAz, I. (1987). Agricultura, ganadería y bosque: la explotación económica de la tierra de Alcaraz (1475-1530). Albacete.

GARCÍA FERNÁNDEZ,J. (1965). "Champs ouverts et champs clôtures en Vieille-Castille". Annales. E.S.C, 20/3-4, 692-718. https://doi.org/10.3406/ahess.1965.421817

García Sanz, A. (1977). Desarrollo y crisis del Antiguo Régimen en Castilla la Vieja. Economía y sociedad en tierras de Segovia de 1500 a 1814. Madrid: Akal.

GARCía SANZ, A. (1998). Los privilegios mesteños en el tiempo, 1273-1836: una revisión de la obra de Julius Klein. En F. Ruiz Martín y A. García Sanz (Eds.), Mesta, trashumancia y lana en la España moderna (pp. 65-89). Barcelona: Crítica y Fundación Duques de Soria.

Gil Cubillo, J. C. (1991). El lobo. Enigmas de un depredador. Madrid: Cairel Ediciones. Goicolea Julián, F. J. (1999). Haro: una villa riojana del linaje Velasco a fines del Medievo. Logroño: Instituto de Estudios Riojanos.

GRAJERA DíAZ, F. (1996). El lobo ibérico en la Baja Extremadura. Badajoz: Universitas. Grande del Brío, R. (1984). El lobo ibérico. Biología y mitología. Madrid: Ed. Hermann Blume.

Grau, J.M.T., Puig, R. y Ruiz-Olmo, J. (1990). Persecución del lobo (Canis lupus L., 1758) en Girona (NE Ibérico) durante los siglos XVIII y XIX: ejemplo de utilización de datos de archivo. Miscelánea Zoológica, 14, 217-223.

HuETZ DE LEMPS, A. (1962). Les terroirs en Vieille Castille et Léon: un type de structure agraire. Annales. E.SC., 17/2, 239-251. https://doi.org/10.3406/ahess.1962.420816 RÖSENER, W. (1990). Los campesinos en la Edad Media. Barcelona: Crítica. 
JiMÉNEZ AlCÁZAR J. F. (1997). Un concejo de Castilla en la frontera de Granada: Lorca 1460-1521. Granada: Universidad de Granada y Ayunt. Lorca.

Kemp, Ch. (1990). Los señores del bosque. Conservación del lobo, el lince, el oso y el bisonte en Europa. Barcelona: Lyns Edicions.

Ladero Quesada, M. A. (1978). España en 1492. Madrid: Ed. Hernando.

LADERO QUESADA, M. A. (1980). La caza en la legislación municipal castellana siglos XIII a XVIII. En la España medieval, (1), 193-222.

Ladero Quesada, M. A. y GonZÁlez Jiménez, M. (1979). Diezmo eclesiástico y producción de cereales en el reino de Sevilla (1408-1503). Sevilla: Universidad de Sevilla.

Le Flem, J. P. (1972). Las cuentas de la Mesta (1510-1709). Moneda y Crédito, 121, 23-104.

Lopez Ontiveros, A., Valle Buenestado, B. y García Verdugo, F. C. (1988). Caza y paisaje geográfico en las tierras Béticas según el Libro de la Montería. En en E. Cabrera (Coord.), Andalucía entre Oriente y Occidente (1236-1492) (pp. 281-308). Córdoba: Diputación de Córdoba.

López VierA, D. (2002). El cazadero real del Lomo del Grullo durante el bajo medievo e inicios de la modernidad: una reserva cinegética de la Corona. En F. J. Pérez-Embid (Coord.), La Andalucía medieval. Actas I Jornadas de Historia Rural y Medio Ambiente (pp. 395-408). Huelva: Universidad de Huelva.

LÓPEZ-SALAZAR PÉREZ, J. (1986). Estructuras agrarias y sociedad rural en La Mancha (siglos XVI-XVII). Ciudad Real: Instituto de Estudios Manchegos.

LuCHíA, C. (2020). 'Por que los montes de esta villa se conserben, e no se disipen como al presente estan': la regulación de los recursos forestales en la corona de Castilla (siglos XIV-XVI). Espacio, Tiempo y Forma. III. Historia Medieval, (33), pp. 303-332.

Marcos Martín, A. (2000). España en los siglos XVI, XVII y XVIII. Economía y sociedad. Barcelona: Ed.Crítica.

Marín Barriguete, F. (1992). Los Reyes Católicos y el Honrado Concejo de la Mesta. Una desmitificación necesaria". Cuadernos de Historia Moderna, (13), 110-141.

Mariño VeIRÁs, D. (1983). Señorío de Santa María de Meira (de 1150 a 1525). Espacio rural, régimen de propiedad y régimen de explotación en la Galicia Medieval. La Coruña.

Martín CEA, J. C. (1991). El mundo rural castellano a fines de la Edad Media. El ejemplo de Paredes de Nava en el siglo XV. Valladolid: Junta de Castilla y León.

MARTín GutiÉRREZ, E. (2003). La identidad rural de Jerez de la Frontera. Territorio y poblamiento durante la baja Edad Media. Cádiz, Universidad de Cádiz.

Martín Martín, J. L. (1990). Evolución de los bienes comunales en el siglo XV. Studia Historica. Historia Medieval, (8), 7-46. 
Menjot, D. (1980). Les murciens du Bas Moyen Âge à la chasse. En La chasse au Moyen Âge: Actes du Colloque Nice [22-24 juin 1979] (pp. 254-273). Paris, les Belles Lettres.

Molenat, J.-P. (1997). Campagnes et monts de Tolede du XIe au XVe siécle. Madrid: Casa de Velázquez.

MOLINIER, A. y MOLINIER-MeYer, N. (1981). Environnement et histoire: les loups et l'homme en France. Revue d'Histoire Moderne et Contemporaine, (28/2), 225-245. https://doi.org/10.3406/rhmc.1981.1140

Monsalvo Antón, J. Ma (1988). El sistema político concejil. El ejemplo del señorío medieval de Alba de Tormes y su concejo de villa y tierra. Salamanca: Universidad de Salamanca.

MontanARI, M. (1984). Vicende di un'espropriazione: il ruolo della caccia nell'economia e nell'alimentazione di ceti rurali. En M. Montanari, Campagne medievale. Strutture produttive, rapporti di lavoro, sistemi alimentari (pp. 174-190). Torino: Einaudi.

Nores, C. y NAVES, J. (1993). Distribución histórica del oso pardo en la península Ibérica. En J. Naves y G. Palomero (Eds.), El oso pardo (Ursus arctos) en España (pp. 13-33). Madrid: ICONA.

NORES Quesada, C. y Liesau, C. (1992). La Zoología Histórica como complemento de la Arqueozoología. El caso de Zebro. Archaeofauna. International Journal of Archaeozoology, (1), 61-71.

Oliva Herrer, R. H. (2002). La Tierra de Campos a fines de la Edad Media. Economía, sociedad y acción política campesina. Valladolid: Universidad de Valladolid.

Ortalli, G. (1997). Natura, storia e mitografia del lupo nel medioevo. En G. Ortalli, Lupi, genti, culture. Uomo e ambiente nel medioevo (pp. 57-122). Turín: Einaudi.

Parejo Delgado, M. J. (1988). Baeza y Úbeda en la baja Edad Media. Granada: Don Quijote.

Pereda HeRnÁndez, J. (1988). Conservación y repoblación de arbolado en Almansa a mediados del siglo XVI. En Actas del I Congreso de Historia de Castilla la Mancha. 7: Conflictos sociales y evolución económica en la Edad Moderna (pp. 8996). Toledo: Juntas de Comunidades de Castilla-La Mancha.

Pino García, J. L. del (1996). Caza y cazadores en la Castilla bajomedieval. Meridies, III, 89-118.

Rey Castelao, O. (1995). Montes y política forestal en la Galicia del Antiguo Régimen. Santiago de Compostela: Universidad de Santiago de Compostela.

Rivero RodríGuez, M. (2005). Caza, monarquía y cultura cortesana. En J. Martínez Millán y S. Fernández Conti (coords.), La monarquía de Felipe II: la Casa del Rey, vol. 1 (pp. 351-376). Madrid: Fundación Mapfre-Tavera.

Rodríguez Molina, J. (1978): El reino de Jaén en la baja Edad Media. Aspectos demográficos y económicos. Granada: Universidad de Granada. 
RODRíGUEZ LlOPIS, M. (1984). Señoríos y feudalismo en el reino de Murcia. Los dominios de la Orden de Santiago entre 1440 y 1515. Murcia: Universidad de Murcia.

Rosas, M. (2009). Dinámica poblacional del lobo (Canis lupus Linnaeus, 1758) en la zona este de la provincia de Castelló de la Plana durante los siglos XVI-XVII (15661624). Galemys, 21/1, 3-15.

Rucquoi, A. (1997). Valladolid en la Edad Media. Genesis de un poder. Valladolid: Junta de Castilla y León.

SÁNCHEZ BENITO, J. Ma (1999). Organización y explotación de la tierra de Huete (siglo XV). Historia. Instituciones. Documentos, (26), 491-546.

SÁNCHEZ CARrera, Ma C. (1997). El bajo Miño en el siglo XV. El espacio y los hombres. La Coruña: Instituto de Estudios Gallegos Padre Sarmiento.

SÁnCHEZ Rubio, Ma A. (1993). El concejo de Trujillo y su alfoz en el tránsito de la Edad Media a la Edad Moderna. Cáceres: Universidad de Extremadura.

Santos Canalejo, E. C. de (1986). La historia medieval de Plasencia y su entorno geohistorico: la Sierra de Béjar y la Sierra de Gredos. Cáceres: Diputación de Cáceres. Sobrado Correa, H. (2003). Los enemigos del campesino. La lucha contra el lobo y otras alimañas nocivas en la Galicia de la Edad Moderna. Obradoiro de Historia Moderna, (12), 105-139.

Solano Ruiz, E. (1978). La Orden de Calatrava en el siglo XV: los señoríos castellanos de la Orden al fin de la Edad Media. Sevilla: Universidad de Sevilla.

SuÁrez Alvarez, Ma J. (1982). La villa de Talavera y su tierra en la Edad Media (13691504). Oviedo: Universidad de Oviedo.

TORQUemada, Ma J. (1997). La protección ecológica en la Castilla bajomedieval. Madrid: UCM.

TORRENTE FERnÁNDEZ, I. (1982). El dominio del monasterio de San Bartolomé de Nava (siglos XIII-XVI). Oviedo: Universidad de Oviedo.

TORres y TAPIA, A. de (1999). Crónica de la Orden de Alcántara. Mérida: Junta de Extremadura y Asamblea de Extremadura (facsímil edic. 1763).

TRISTÁn García, F. (2000). 'Va el lobo'. Aproximación histórica al depredador en la tierra de Baza en la alta edad moderna. Chronica Nova, (27), 239-281. 